\title{
Análise do pH e da atividade antimicrobiana de um novo medicamento intracanal biocerâmico Bio-C Temp
}

\author{
Analysis of pH and antimicrobial activity of a new bioceramic intracanal medicament Bio-C Temp \\ Análisis de pH y actividad antimicrobiana de un nuevo fármaco intracanal biocerámico Bio-C
}

Temp

Recebido: 26/05/2021 | Revisado: 04/06/2021 | Aceito: 09/06/2021 | Publicado: 23/06/2021

Francisca Lívia Parente Viana

ORCID: https://orcid.org/0000-0002-6762-0242

Universidade Federal do Ceará, Brasil

E-mail: liviapviana@ hotmail.com

Marcelo Bonifácio da Silva Sampieri

ORCID: https://orcid.org/0000-0001-7942-6642

Universidade Federal do Ceará, Brasil

E-mail: mar_sampieri@hotmail.com

Priscilla Parente Viana

ORCID: https://orcid.org/0000-0003-4382-8142

Universidade Federal do Ceará, Brasil

E-mail: priscilla_parente@hotmail.com

Suyane Maria Luna Cruz

ORCID: https://orcid.org/0000-0002-2337-6575

Universidade Federal do Ceará, Brasil

E-mail: suyaneluna@yahoo.com.br

Rodrigo Ricci Vivan

ORCID: https://orcid.org/0000-0002-0419-5699

Universidade de São Paulo, Brasil

E-mail: Rodrigo.vivan@fob.usp.br

Marco Antônio Húngaro Duarte

ORCID: https://orcid.org/0000-0003-3051-737X

Universidade de São Paulo, Brasil

E-mail: mhungaro@fob.usp.br

Iriana Carla Junqueira Zanin

ORCID: https://orcid.org/0000-0001-5271-9808

Universidade Federal do Ceará, Brasil E-mail: irianazanin@gmail.com

Bruno Carvalho de Vasconcelos

ORCID: https://orcid.org/0000-0003-4415-5680

Universidade Federal do Ceará, Brasil

E-mail: bcv@ufc.br

\begin{abstract}
Resumo
As medicações intracanais com atividade antimicrobiana são importantes como coadjuvantes na desinfecção do sistema de canais radiculares. Diante disso, esse estudo avaliou, in vitro, o $\mathrm{pH}$ e a atividade antimicrobiana de um novo medicamento intracanal biocerâmico (Bio-C Temp) em comparação com medicações a base de hidróxido de cálcio (HC). A determinação do $\mathrm{pH}$ foi realizada por meio de um pHmetro nos períodos de 30 minutos, 3, 24, $72 \mathrm{e}$ 168 horas. As medicações Ultracal ${ }^{\circledR} \mathrm{XS}$, Hidróxido de cálcio com soro (HC/Soro), Hidróxido de cálcio com propilenoglicol (HC/PG) e Bio-C Temp (biocerâmico) foram testadas contra biofilmes de duas cepas de Enterococcus faecalis (ATCCs 29212 e 4083) usando os testes de contato direto (TCD) e restrito por membrana (TRM). A sobrevivência bacteriana foi determinada pela contagem das unidades formadoras de colônias (UFCs) e os dados foram comparados estatisticamente $(\mathrm{P}<0,05)$. A pasta Bio-C Temp apresentou o menor valor de $\mathrm{pH}$ nos tempos avaliados. Os grupos HC/Soro e HC/PG apresentaram maior ação frente ao controle no TCD $(\mathrm{P}<0,05)$ contra as duas cepas testadas. Ainda, o HC/PG mostrou maior atividade antibacteriana que o Bio-C Temp $(\mathrm{P}<0,05)$. Todos os grupos apresentaram diferença ante o grupo controle no TRM contra a cepa 29212, e somente o HC/PG mostrou diferença significativa frente ao controle contra biofilmes da cepa 4083. O Bio-C Temp mostrou ação antibacteriana similar ao Ultracal ${ }^{\circledR} \mathrm{XS}$, no entanto apresentou menor ação em relação ao HC/PG. A pasta de hidróxido de cálcio associada com propilenoglicol mostrou vantagens em relação às demais testadas.
\end{abstract}

Palavras-chave: Hidróxido de cálcio; Silicato de cálcio; Enterococcus faecalis; Ação antimicrobiana. 


\begin{abstract}
Intracanals medications with antimicrobial activity are important as adjuvants in disinfecting the root canal system. Therefore, this study evaluated, in vitro, the $\mathrm{pH}$ and antimicrobial activity of a new bioceramic intracanal medication (Bio-C Temp) compared to calcium hydroxide (HC) medications. The determination of $\mathrm{pH}$ was carried out by means of a pH meter in the periods of 30 minutes, $3,24,72$ and 168 hours. The medications Ultracal ${ }^{\circledR} \mathrm{XS}, \mathrm{Calcium}$ hydroxide with saline (HC/Saline), Calcium hydroxide with propyleneglycol (HC/PG) and Bio-C Temp (bioceramic) were tested against biofilms of two strains of Enterococcus faecalis (ATCCs 29212 and 4083) using direct contact (DCT) and membrane restricted (MRT) tests. Bacterial survival was determined by counting colony-forming units (UFCs) and the data were compared statistically $(\mathrm{P}<0.05)$. The Bio-C Temp paste showed the lowest $\mathrm{pH}$ value in the evaluated times. The HC/Saline and HC/PG groups showed greater action compared to the control in the DCT $(\mathrm{P}<0.05)$ against the two strains tested. In addition, HC/PG showed greater antibacterial activity than Bio-C Temp $(\mathrm{P}<0.05)$. All groups showed a difference compared to the control group in the MRT against strain 29212, and only HC/PG showed a significant difference compared to the control against biofilms of strain 4083. Bio-C Temp showed antibacterial action similar to Ultracal ${ }^{\circledR} \mathrm{XS}$, however showed less action in relation to HC/PG. The calcium hydroxide paste associated with propylene glycol showed advantages over the others tested.
\end{abstract}

Keywords: Calcium hydroxide; Calcium silicate; Enterococcus faecalis; Antimicrobial action.

\title{
Resumen
}

Los medicamentos intracanal con actividad antimicrobiana son importantes como adyuvantes en la desinfección del sistema de conductos radiculares. Por lo tanto, este estudio evaluó, in vitro, el $\mathrm{pH}$ y la actividad antimicrobiana de un nuevo medicamento intracanal biocerámico (Bio-C Temp) en comparación con medicamentos de hidróxido de calcio (HC). La determinación del $\mathrm{pH}$ se realizó mediante un medidor de $\mathrm{pH}$ en los periodos de 30 minutos, 3, 24, 72 y 168 horas. Los medicamentos Ultracal ${ }^{\circledR} \mathrm{XS}$, hidróxido de calcio con suero (HC/suero), hidróxido de calcio con propilenglicol (HC/PG) y Bio-C Temp (biocerámica) se probaron contra biopelículas de dos cepas de Enterococcus faecalis (ATCC 29212 y 4083) utilizando pruebas de contacto directo (TCD) y de membrana restringida (TRM). La supervivencia bacteriana se determinó contando las unidades formadoras de colonias (UFCs) y los datos se compararon estadísticamente $(\mathrm{P}<0,05)$. La pasta Bio-C Temp tuvo el valor de $\mathrm{pH}$ más bajo en los tiempos evaluados. Los grupos HC/Soro y HC/PG mostraron una mayor acción en comparación con el control en el TCD ( $<<0.05)$ contra las dos cepas probadas. Además, HC/PG mostró una mayor actividad antibacteriana que Bio-C Temp ( $\mathrm{P}<$ 0,05). Todos los grupos mostraron una diferencia en comparación con el grupo de control en el TRM contra la cepa 29212, y solo HC/PG mostró una diferencia significativa en comparación con el control contra las biopelículas de la cepa 4083. Bio-C Temp mostró una acción antibacteriana similar a Ultracal ${ }^{\circledR} \mathrm{XS}$, sin embargo mostró menor acción en relación a HC/PG. La pasta de hidróxido de calcio asociada con propilenglicol mostró ventajas sobre las otras probadas.

Palabras clave: Hidróxido de cálcio; Silicato de cálcio; Enterococcus faecalis; Acción antimicrobiana.

\section{Introdução}

A limpeza e modelagem do sistema de canais radiculares (SCR) visam reduzir ao máximo o número de microrganismos e seus subprodutos do interior dos canais. No entanto, a complexidade anatômica radicular torna-se um obstáculo para a correta antissepsia, levando a uma permanência de bactérias em locais que não são tocados durante o preparo químico-mecânico, podendo levar ao insucesso do tratamento (Zancan, et al., 2016). Diante disso, o uso de medicação intracanal é de extrema importância como coadjuvante na desinfecção do sistema de canais radiculares.

Diversas substâncias são descritas na literatura para esse fim, sendo o hidróxido de cálcio (HC) o mais utilizado devido suas propriedades antimicrobianas. A eficácia do HC está relacionada a liberação de íons hidroxila, que reagem com várias biomoléculas causando dano a membrana citoplasmática e desnaturação protéica dos microrganismos (Mohammadi \& Dummer, 2011); além de aumentar o nível de pH (Kim \& Kim, 2014). Outra propriedade importante do HC é sua capacidade de promover a inativação de lipopolissacarídeos bacterianos encontrados na membrana externa de bactérias gram-negativas (Tanomaru, et al., 2003). Apesar do sucesso do hidróxido de cálcio como medicação intracanal, várias espécies microbianas, como o Enterococcus faecalis, apresentam resistência para o pH alcalino (Nakajo, et al., 2006; Ferreira, et al., 2007). Além disso, a capacidade desse microrganismo de aderir, colonizar e formar biofilmes favorece associações entre espécies, aumentando sua resistência a substâncias antimicrobianas (Guerreiro-Tanomaru, et al., 2013). A associação do HC com veículos de diferentes características físico-químicas visam facilitar o manuseio clínico e obter sinergismo de ação 
antimicrobiana, interferindo na velocidade de dissociação iônica, solubilidade e difusibilidade, exercendo uma influência direta sobre o pH (De Andrade Ferreira, et al., 2004) e sobre as atividades biológicas (Fava \& Saunders, 1999; Zancan, et al., 2016).

Os materiais endodônticos biocerâmicos à base de silicatos e/ou fosfato de cálcio atraíram considerável atenção devido às suas excelentes propriedades físico-químicas e biológicas (Al-Haddad \& Aziz, 2016). O crescente interesse por esses materiais é evidenciado pelo desenvolvimento de novos medicamentos bioativos à base de silicato de cálcio, como o Bio-C Temp (Angelus Indústria de Produtos Odontológicos S/A, Londrina, PR, Brasil). Esse medicamento se apresenta como uma pasta pronta para uso, é biocompatível, possui pH alcalino e boa radiopacidade (Villa, et al., 2020). Além disso, o Bio-C Temp induz a biomineralização e possui atividade antimicrobiana (Guerreiro, et al., 2021).

Diante da importância de uma medicação intracanal apresentar alta alcalinidade proporcionando uma melhor atividade antimicrobiana, este estudo tem como objetivo avaliar o $\mathrm{pH}$ e a atividade antimicrobiana de um novo medicamento biocerâmico (Bio-C Temp) em comparação aos medicamentos à base de hidróxido de cálcio (Ultracal ${ }^{\circledR} \mathrm{XS}$, HC/Soro e HC/Propilenoglicol) por meio dos testes de contato direto e restrito por membrana contra biofilmes de duas espécies de Enterococcus faecalis. A hipótese nula testada é que todas as pastas apresentarão similaridade em relação ao pH e a atividade antimicrobiana.

\section{Metodologia}

\subsection{Medicações intracanais}

As medicações intracanais avaliadas, seus fabricantes e composições estão descritos na Tabela 1. As formulações $1 \mathrm{e}$ 2 são comercialmente apresentadas pronto para uso. As formulações utilizadas nos grupos 3 e 4 foram manipuladas agregando o pó do hidróxido de cálcio ao líquido, até a obtenção de uma forma pastosa e homogênea.

Tabela 1: Medicamentos intracanais, fabricantes e composições.

\begin{tabular}{|c|c|l|l|}
\hline Grupos & Medicação & \multicolumn{1}{|c|}{ Fabricante } & \multicolumn{1}{|c|}{ Composição } \\
\hline G1 & Ultracal ${ }^{\circledR}$ XS & $\begin{array}{l}\text { Ultradent Products Inc., South Jordan, } \\
\text { UT, USA }\end{array}$ & $\begin{array}{l}\text { Hidróxido de cálcio } \\
\text { Sulfato de bário } \\
\text { Metilcelulose } \\
\text { Solução aquosa }\end{array}$ \\
\hline G2 & Bio-C Temp & $\begin{array}{l}\text { Angelus Indústria de Produtos } \\
\text { Odontológicos S/A, Londrina, PR, Brasil }\end{array}$ & $\begin{array}{l}\text { Silicato de cálcio } \\
\text { Aluminato de cálcio } \\
\text { Óxido de cálcio } \\
\text { Tungstato de cálcio } \\
\text { Óxido de titânio }\end{array}$ \\
\hline G3 & HC/Soro & $\begin{array}{l}\text { Biodinâmica Química e Farmacêutica } \\
\text { Ltda, Ibiporã, PR, Brasil }\end{array}$ & $\begin{array}{l}\text { Hidróxido de cálcio } \\
\text { Soro fisiológico }\end{array}$ \\
\hline G4 & HC/PG & $\begin{array}{l}\text { Biodinâmica Química e Farmacêutica } \\
\text { Ltda, Ibiporã, PR, Brasil }\end{array}$ & $\begin{array}{l}\text { Hidróxido de cálcio } \\
\text { Propilenoglicol }\end{array}$ \\
\hline
\end{tabular}

Fonte: Autores.

\subsection{Avaliação do PH}

Foram utilizados tubos de polietileno com diâmetro interno de $1,0 \mathrm{~mm}$ e comprimento de $1 \mathrm{~cm}$ com apenas uma de suas extremidades fechada. Os espécimes $(n=10)$ deste ensaio foram obtidos por meio da inserção das pastas no interior dos tubos com auxílio de calcadores do tipo Paiva até seu completo preenchimento. Após o preparo, cada espécime foi imerso em tubo Falcon estéril e livre de DNAse e RNAse (Techno Plastic Products Ag., Trasadingen, Suíça) contendo 10 mL de água deionizada e mantidos na estufa à $37^{\circ} \mathrm{C}$ e $100 \%$ de humidade por todo o período experimental. As avaliações foram realizadas nos períodos de 30 minutos, 3, 24, 72 e 168 horas, nos quais, a cada período, os espécimes foram cuidadosamente colocados em um novo tubo com o mesmo volume de água deionizada. A determinação do $\mathrm{pH}$ foi realizada por meio de um $\mathrm{pHmetro}$ 
previamente calibrado com soluções de pH conhecido (4, 7 e 10). Os tubos Falcon, após a remoção dos espécimes, foram agitados durante 5 segundos e realizada a leitura.

\subsection{Microrganismos}

Foram utilizadas duas cepas de bactérias gram-positivas e facultativas de Enterococcus faecalis: ATCC 29212 (cepa isolada do trato geniturinário) e ATCC 4083 (cepa isolada de canal radicular de dente necrosado). Os estoques bacterianos foram conservados em meio Brain Heart Infusion (BHI; Kasvi, São José dos Pinhais, PR, Brasil) com glicerol a 20\% em freezer $-80^{\circ} \mathrm{C}$. Para reativá-las foram realizados repiques para viabilização das cepas bacterianas. Foram inoculados $200 \mu \mathrm{L}$ de cada cepa em frascos contendo $10 \mathrm{~mL}$ de caldo $\mathrm{BHI}$, em duplicata e incubados à $37^{\circ} \mathrm{C}$ por 18 horas. A pureza da cultura foi verificada e o inóculo bacteriano foi padronizado para uma densidade óptica de 0,1 (620 nm), para uma turbidez equivalente a um crescimento de $1-2 \times 108 \mathrm{UFC} / \mathrm{mL}$.

\subsection{Atividade antimicrobiana - Teste de Contato Direto e Restrito por Membrana}

A formação de biofilmes foi induzida em membranas de nitrato de celulose de $13 \mathrm{~mm}$ de diâmetro e poros de $0,22 \mu \mathrm{m}$ (Adria laboratórios, Londrina, Paraná). As membranas foram colocadas sobre a superfície de placas petri contendo ágar BHI. Em seguida, alíquotas de $40 \mu \mathrm{L}$ da suspensão bacteriana foram aplicadas às superfícies das membranas e as placas armazenadas durante 48 horas a $37^{\circ} \mathrm{C}$.

Para o teste de contato direto (TCD) material/biofilme, 0,15 $\mathrm{g}$ da pasta fresca foi colocada sobre o biofilme formado sobre as membranas. No teste restrito por membrana (TRM), uma membrana de nitrato de celulose estéril foi interposta entre o biofilme e a pasta. Foram utilizadas três membranas para cada pasta, com seis repetições em ocasiões distintas. O tempo de contato para ambos os testes foi de 30 minutos a $37^{\circ} \mathrm{C}$ (Barros, et al., 2014). Após o período de contato, a pasta foi removida da superfície da membrana no contato direto. No teste indireto, a membrana de celulose com a pasta foi descartada. Em ambas as condições de contato, grupos-controle foram preparados utilizando uma membrana com biofilme formado sem qualquer contato com os materiais. As membranas foram transferidas para um frasco contendo $2 \mathrm{~mL}$ de solução salina estéril que foram levemente agitados para remover células fracamente aderidas. Em seguida foram transferidas para outro frasco contendo $2 \mathrm{~mL}$ de solução salina e agitados por vórtex durante 1 minuto $(30 s+15 s+15 s)$ intercalado em banho de gelo. Foi realizada uma série de dez diluições em solução salina e alíquotas de $10 \mu \mathrm{L}$ de cada diluição foram plaqueadas em ágar BHI. As unidades formadoras de colônia (UFC) foram contadas após incubação por 24 horas a $37^{\circ} \mathrm{C}$ e a transformação $\operatorname{logarítmica~}\left(\log { }^{10}\right)$ dos dados foi realizada.

\subsection{Análise Estatística}

Os dados de ambas as avaliações foram tabulados e submetidos ao teste Shapiro-Wilks para determinação de sua normalidade, o qual apontou natureza não paramétrica. Desta forma, foram empregados a análise de variância de KruskalWallis e o teste de comparações múltiplas de Dunn para análise entre as diferentes medicações no pH e nos testes de contato. A análise de Mann-Whitney foi utilizada para comparações entre as cepas testadas. Todos com significância estabelecida em $5,0 \%$.

\section{Resultados}

Os resultados desse estudo mostram que o BioC-Temp apresentou os menores valores de $\mathrm{pH}$, mostrando diferença significante com as pastas de HC/Soro e HC/PG nos tempos $30 \mathrm{~min}$, 24h, 72h e 168h (P < 0,05). A pasta HC/PG apresentou o maior valor de $\mathrm{pH}$ até o período de $24 \mathrm{~h}$ em relação aos outros medicamentos. A pasta HC/Soro apresentou maior valor de $\mathrm{pH}$ 
no período de 7 dias. A Tabela 2 mostra os resultados do $\mathrm{pH}$ nos tempos avaliados.

Tabela 2. Médias (valores mínimos e máximos) de pH encontrados nos diferentes períodos.

\begin{tabular}{cccccc}
\hline \multirow{2}{*}{ Medicações } & 30 min. & $\mathbf{3}$ hrs. & $\mathbf{2 4}$ hrs. & $\mathbf{7 2 ~ h r s . ~}$ & $\mathbf{1 6 8}$ hrs. \\
\cline { 2 - 6 } & Média (mín. - máx.) & Média (mín. - máx.) & Média (mín. - máx.) & Média (mín. - máx.) & Média (mín. - máx.) \\
\hline Ultracal ${ }^{\circledR}$ XS & $10.19^{\mathrm{bc}}(9.9-10.7)$ & $10.5^{\mathrm{a}}(8.3-10.7)$ & $10.43^{\mathrm{ac}}(10.2-10.7)$ & $10.36^{\mathrm{ab}}(9.5-11.2)$ & $9.63^{\mathrm{ab}}(8.7-11.0)$ \\
Bio-C Temp & $9.49^{\mathrm{b}}(9.2-9.8)$ & $8.93^{\mathrm{b}}(8.4-9.3)$ & $9.78^{\mathrm{a}}(9.5-10.1)$ & $9.26^{\mathrm{b}}(8.6-10.4)$ & $8.88^{\mathrm{b}}(7.9-10.6)$ \\
HC/Soro & $10.7^{\mathrm{a}}(10.2-10.9)$ & $9.94^{\mathrm{ab}}(9.1-10.5)$ & $10.75^{\mathrm{bc}}(10.4-11.1)$ & $10.74^{\mathrm{a}}(10.0-11.4)$ & $10.08^{\mathrm{a}}(8.2-11.1)$ \\
HC/PG & $10.72^{\mathrm{ac}}(10.4-11.2)$ & $10.52^{\mathrm{a}}(10.0-1.4)$ & $10.96^{\mathrm{b}}(10.5-11.2)$ & $10.69^{\mathrm{a}}(8.7-11.3)$ & $9.94^{\mathrm{a}}(8.0-10.8)$ \\
\hline
\end{tabular}

a,b Letras diferentes sobrescritas indicam diferenças significantes de acordo com os testes de Kruskal-Wallis e teste de comparação múltipla de Dunn $(\mathrm{P}<0,05)$. Fonte: Autores.

Os resultados encontrados nos testes de contato direto e restrito por membrana encontram-se no Gráfico 1. A Tabela 3 mostra os dados relativos a atividade antimicrobiana das pastas intracanais testadas contra biofilmes de E. faecalis em TCD. Os resultados mostram que as pastas HC/Soro e HC/PG apresentaram maior ação antimicrobiana com diferença significante em relação ao grupo controle nas duas cepas testadas $(\mathrm{P}<0,05)$. Ainda, a pasta HC/PG apresentou maior ação que a pasta BioC Temp $(\mathrm{P}<0,05)$ considerando a cepa 29212.

Gráfico 1. Medianas do crescimento bacteriano $\left(\log ^{10}\right)$ após testes de contato direto e restrito por membrana.

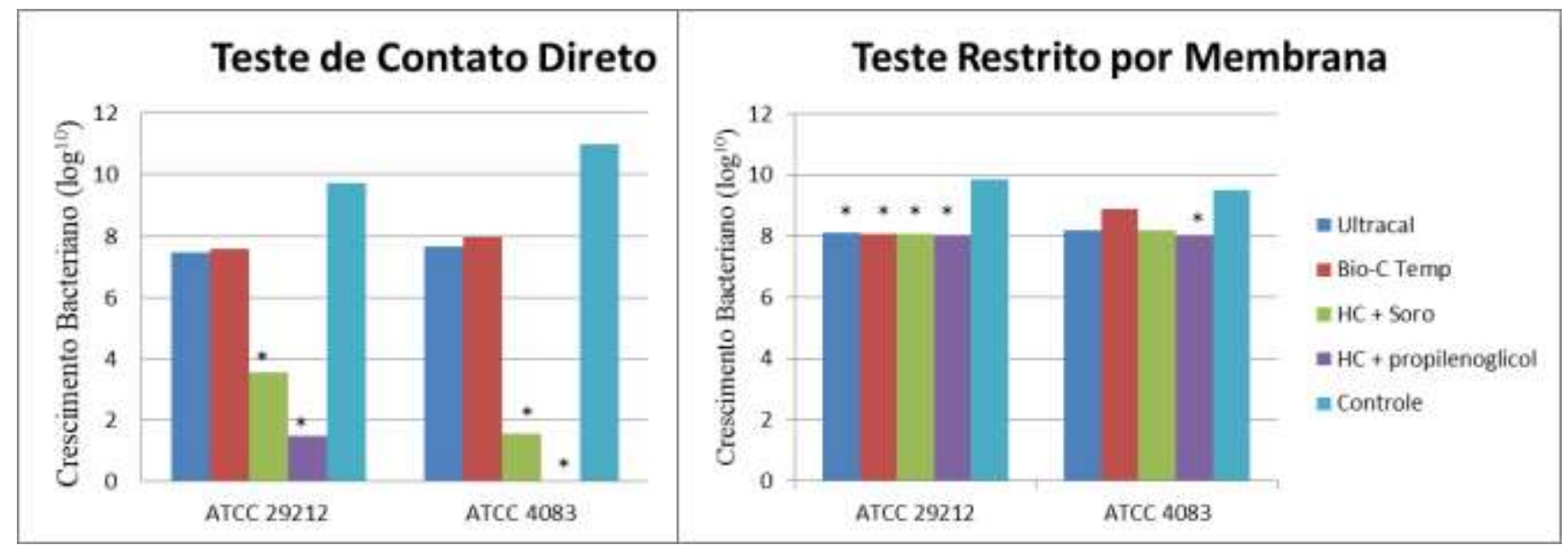

* Indicam diferenças significantes contra o grupo controle de acordo com os testes de Kruskal-Wallis e teste de comparação múltipla de Dunn $(P<0,05)$. Fonte: Autores. 
Tabela 3. Medianas (valores míimos e máximos) do crescimento bacteriano $\left(\log ^{10}\right)$ após contato direto com os biofilmes bacterianos.

\begin{tabular}{|c|c|c|}
\hline \multirow{2}{*}{ Medicaçãa } & ATCC 29212 & ATCC 4083 \\
\hline & Mediana (mín. - máx.) & Mediana (mín. - máx.) \\
\hline Ultracal ${ }^{\circledR} \mathrm{XS}$ & $7,46^{\mathrm{abc}, \mathrm{A}}(5,15-7,91)$ & $7,67 \mathrm{ab}, \mathrm{A}(6,67-9,06)$ \\
\hline Bio-C Temp & $7,57 \mathrm{ab}, \mathrm{A}(7,04-9,02)$ & $7,95^{\mathrm{ab}, \mathrm{A}}(5,59-8,07)$ \\
\hline HC/Soro & $3,56^{\mathrm{bc}, \mathrm{A}}(0-4,66)$ & $1,58^{\mathrm{b}, \mathrm{A}}(0-3,80)$ \\
\hline $\mathrm{HC} / \mathrm{PG}$ & $1,50^{\mathrm{c}, \mathrm{A}}(0-3,56)$ & $0^{\mathrm{b}, \mathrm{A}}(0-3,68)$ \\
\hline Controle & $9,74^{\mathrm{a}, \mathrm{A}}(9,37-12,10)$ & $10,99^{\text {a, }}$ A $(7,85-12,60)$ \\
\hline
\end{tabular}

a,bLetras diferentes sobrescritas indicam diferenças significantes de acordo com os testes de Kruskal-Wallis e teste de comparação múltipla de Dunn $(P<0,05)$.

$\mathrm{A}, \mathrm{B}$ Letras maiúsculas diferentes sobrescritas indicam diferenças significantes de acordo com o teste de Mann-Whitney $(\mathrm{P}<0,05)$ entre as cepas testadas.

Fonte: Autores.

Os resultados do teste restrito por membrana (Tabela 4) mostram que todos os grupos de medicações apresentaram uma redução significativa da contagem bacteriana $(\mathrm{P}<0,05)$ frente ao grupo controle considerando a cepa genito-urinária (ATCC 29212). Apenas o grupo HC/PG mostrou diferença significante ante ao controle contra biofilmes da cepa endodôntica (ATCC 4083).

Tabela 4. Medianas (valores mínimos e máximos) do crescimento bacteriano $\left(\log ^{10}\right)$ após contato indireto com os biofilmes bacterianos.

\begin{tabular}{|c|c|c|}
\hline \multirow{2}{*}{ Medicações } & ATCC 29212 & ATCC 4083 \\
\hline & Mediana (mín. - máx.) & Mediana (mín. - máx.) \\
\hline Ultracal ${ }^{\circledR} \mathrm{XS}$ & $8,10^{\mathrm{b}, \mathrm{A}}(7,88-8,24)$ & $8,20^{\mathrm{ab}, \mathrm{A}}(8,12-9,02)$ \\
\hline Bio-C Temp & $8,08^{\mathrm{b}, \mathrm{A}}(7,65-8,27)$ & $8,88^{a b, ~ A ~}(7,03-9,22)$ \\
\hline HC/Soro & $8,07^{\mathrm{b}, \mathrm{A}}(7,97-8,18)$ & 8,18 ab, A $(7,99-9,01)$ \\
\hline $\mathrm{HC} / \mathrm{PG}$ & $8,04^{\mathrm{b}, \mathrm{A}}(7,79-9,13)$ & $8,03^{\mathrm{b}, \mathrm{A}}(6,65-8,81)$ \\
\hline Controle & $9,84^{\text {a, }, \mathrm{A}}(8,85-10,02)$ & $9,48^{\mathrm{a}, \mathrm{A}}(8,84-9,80)$ \\
\hline
\end{tabular}

${ }^{\mathrm{a}, \mathrm{b}}$ Letras diferentes sobrescritas indicam diferenças significantes de acordo com os testes de Kruskal-Wallis e teste de comparação múltipla de Dunn $(P<0,05)$.

A,B Letras maiúsculas diferentes sobrescritas indicam diferenças significantes de acordo com o teste de Mann-Whitney $(\mathrm{P}<0,05)$ entre as cepas testadas.Fonte: Autores.

Avaliando os resultados encontrados nas mesmas condições de contato comparando as cepas de E. faecalis, a análise estatística constatou que não houve diferença significante para nenhum grupo.

\section{Discussão}

O presente estudo avaliou o $\mathrm{pH}$ e a atividade antimicrobiana de um novo medicamento intracanal biocerâmico (Bio-C Temp) em comparação aos medicamentos à base de hidróxido de cálcio (Ultracal ${ }^{\circledR} \mathrm{XS}, \mathrm{HC} / \mathrm{Soro}$ e HC/Propilenoglicol) por 
meio dos testes de contato contra biofilmes de duas espécies de Enterococcus faecalis. Considerando os resultados encontrados, rejeitou-se a hipótese nula inicialmente sugerida, uma vez que algumas pastas apresentaram ação antimicrobiana diferentes entre si e superiores ao grupo controle. Os materiais biocerâmicos são caracterizados por um $\mathrm{pH}$ alcalino e atividade antibacteriana, e são quimicamente estáveis em um ambiente biológico (Candeiro, et al., 2012, Simundi'c Muniti'c, et al., 2019). Devido aos poucos estudos encontrados acerca do Bio-C Temp, verificou-se a necessidade de uma melhor avaliação de suas propriedades antimicrobianas. As pastas de HC continuam sendo a medicação de escolha para uso entre sessões por reduzirem o número de microrganismos do sistema de canais radiculares devido a sua ação biológica (Bystrom, Claesson \& Sundqvist, 1985; Peters, et al., 2005; Zancan, et al., 2016). Para utilização do HC como medicação intracanal, é necessária a adição de um veículo, pois este determina a taxa de dissociação iônica e difusão desses íons através da dentina (Mohammadi \& Dummer, 2011), contribuindo com a ação antimicrobiana. Esses veículos podem ser aquosos, viscosos ou oleosos. Nesse estudo avaliamos a pasta de $\mathrm{HC}$ com um veículo aquoso (soro), um viscoso (propilenoglicol) e uma pasta pronta para uso (Ultracal ${ }^{\circledR} \mathrm{XS}$ ), além da pasta com base de silicato de cálcio (Bio-C Temp).

A capacidade antimicrobiana dos medicamentos intracanais é afetada pela velocidade de sua desintegração em íons cálcio e hidroxila, elevando o pH do meio (Mohammadi \& Dummer, 2011; Tabrizizadeh, et al., 2015). Através da peroxidação de lipídios, os íons hidroxila aumentam a permeabilidade da membrana bacteriana, a desnaturação de proteínas e a inativação de enzimas celulares (Mohammadi \& Dummer, 2011; Tabrizizadeh, et al., 2015), causando um efeito letal sobre as bactérias. Um valor de pH alcalino (8,6 a 10,3) é necessário para que ocorra uma ação biológica das medicações intracanais (Estrela, et al., 1999). No entanto, um nível de $\mathrm{pH}$ acima de 11 pode resultar em uma maior citotoxicidade no tecido periapical (Rehman, et al., 1996). Nesse estudo, todos os grupos avaliados apresentaram o pH dentro dos limites aceitáveis. Os grupos HC/PG, HC/Soro e o Ultracal ${ }^{\circledR}$ XS mostraram valores de pH similares a outros estudos (Carvalho, et al., 2016; Pereira, et al., 2019). Já o Bio-C Temp apresentou os menores valores de $\mathrm{pH}$ em todos os períodos experimentais com diferença significativa com os grupos HC/Soro e HC/PG (P < 0,05). Os materiais a base de silicatos de cálcio, quando hidratados, formam o hidróxido de cálcio que se dissociam rapidamente em íons $\mathrm{Ca}^{++} \mathrm{e} \mathrm{OH}^{-}$aumentando o $\mathrm{pH}$ do meio (Villa, et al., 2020). No entanto, a baixa solubilidade do $\mathrm{HC}$ pode ser responsável pela lenta liberação dos íons $\mathrm{OH}^{-}$, justificando os menores valores do pH do Bio-C Temp. Alguns estudos reportam um tempo mínimo de 14 dias de alcalinização ideal para as medicações intracanais, influenciando na sua atividade antimicrobiana (Nerwich, et al., 1993; Pereira, et al., 2019). No entanto, os medicamentos de hidróxido de cálcio apresentam rápido efeito alcalino após 10 minutos de contato com o meio, resultando em uma liberação instantânea de íons hidroxila, o que justifica os tempos avaliados nesse estudo (Zemner, et al., 2007; Carvalho, et al., 2016).

Apesar do conhecimento do perfil multiespécie da infecção endodôntica, as avaliações dos biofilmes monoespécies de E. faecalis são fundamentais, pois esses microrganismos são mais resistentes à restrição de nutrientes na coexistência com outras espécies presentes nas infecções endodônticas (Gao, et al., 2016), mostrando maior sobrevida. A cepa de E. faecalis mais utilizada nos estudos in vitro é a ATCC 29212, com origem no trato geniturinário (Kangarlou, et al., 2016; Collares, et al., 2017; Cheng, et al., 2017; Swimberghe, et al., 2018), no entanto, o uso de cepas isoladas do canal radicular é recomendado, já que diferentes cepas da mesma espécie podem diferir devido a características adquiridas (Wang, et al., 2014; Barros, et al., 2014; Zancan, et al., 2018). Diante disso, optou-se pela inclusão de uma cepa isolada de canais radiculares necrosados (ATCC 4083) (Zancan, et al., 2018; Oda, et al., 2019). Nesse estudo, não houve diferença significante com os resultados encontrados comparando-se as cepas. Porém, podemos observar que as pastas HC/Soro e HC/PG mostraram maior redução da contagem bacteriana no TCD contra a cepa endodôntica. Esse resultado também foi encontrado por Zancan et al. (2018), que mostram que a cepa isolada do trato urinário é mais resistente que aquela isolada do canal radicular e se comporta de maneira diferente em relação a resistência de medicamentos.

Nesse estudo, todas as pastas promoveram uma redução das contagens bacterianas dos biofilmes de E. faecalis nas 
duas condições de contato, mas nem todas foram significativas. Em relação ao TCD, as pastas HC/Soro e HC/PG apresentaram maior ação antimicrobiana contra as duas cepas testadas $(\mathrm{P}<0,05)$. Além disso, a pasta HC/PG mostrou uma maior ação que a pasta Bio-C Temp ( < 0,05) considerando biofilmes da cepa 29212. O veículo utilizado nas pastas obturadoras pode influenciar o efeito antibacteriano de um medicamento por meio da velocidade de dissociação iônica e difusão pelos tecidos periapicais. Como o propilenoglicol apresenta menor tensão superficial que a água, ele permite uma maior difusão do medicamento através da membrana celular das bactérias do biofilme (Pereira, et al., 2019). O Ultracal ${ }^{\circledR} X S$ é uma pasta pronta para uso que apresenta na sua composição $35 \%$ de HC, $2 \%$ de sulfato de bário, água e metilcelulose. Apesar de ser constituída de $\mathrm{HC}$ com veículo aquoso, não apresentou resultados semelhantes ao grupo HC/Soro. Essa diferença pode estar relacionada com a baixa concentração de $\mathrm{HC}$ na pasta Ultracal ${ }^{\circledR} \mathrm{XS}$, promovendo uma menor liberação de íons nos tecidos e, consequentemente, menor ação antimicrobiana. A pasta Bio-C Temp também mostrou baixa ação antimicrobiana, o que pode estar relacionada a sua baixa alcalinidade e a menor formação de moléculas de HC na reação de hidratação (Guerreiro, et al., 2021). Nesse teste, o Bio-C Temp e Ultracal ${ }^{\oplus}$ XS tiveram ação antimicrobiana semelhantes.

Sabe-se que na condição do contato indireto (TRM), a existência de uma barreira com capacidade de limitar o contato entre os materiais e o biofilme sugere a necessidade de agentes solúveis com capacidade de difundirem-se pela umidade do próprio material e do biofilme. Nesta condição, apenas o grupo HC/PG mostrou uma maior ação antimicrobiana com diferença significante em relação ao grupo controle contra as duas cepas $(\mathrm{P}<0,05)$. Esse bom resultado pode estar relacionado ao veículo utilizado, que promoveu uma maior dissociação iônica, favorecendo a difusão dos íons hidroxila através da membrana, proporcionando uma maior efetividade. Os outros medicamentos tiveram resultados semelhantes.

As limitações deste estudo in vitro incluem o uso de biofilmes monoespécies de E. faecalis, que são provavelmente mais suscetíveis do que biofilmes multiespécies. Apesar do conhecimento do perfil polimicrobiano das infecções endodônticas, a maioria dos estudos in vitro utilizou biofilmes monoespécies para testar materiais endodônticos, o que possibilitou a comparação de nossos dados com estudos anteriores (Barros, et al., 2014; Tabrizizadeh, et al., 2015; Collares, et al., 2018; Balto, et al., 2020; Guerreiro, et al., 2021).

\section{Conclusão}

As medicações intracanais devem apresentar elevada capacidade antimicrobiana, principalmente contra biofilmes, colaborando com a sanificação do SCR e com a manutenção da descontaminação alcançada durante o preparo químicomecânico. De acordo com os resultados encontrados nesse estudo, o Bio-C Temp apresentou pH alcalino e ação antibacteriana semelhante ao Ultracal ${ }^{\circledR} \mathrm{XS}$; no entanto apresentou menor ação em relação ao HC/PG. A pasta de hidróxido de cálcio associada com propilenoglicol mostrou vantagens em relação às demais testadas, apresentando os maiores valores de $\mathrm{pH}$ nos tempos avaliados e maior ação antimicrobiana contra as duas cepas de E. faecalis. Porém, diante das limitações desse estudo, sugere-se uma melhor avaliação acerca das propriedades do Bio-C Temp.

\section{Referências}

Al-Haddad, A., \& Che Ab Aziz, Z. A. (2016). Bioceramic-based root canal sealers: a review. Int J Biomater, 9753210.

Balto, H., Bukhary, S., Al-Omran, O., BaHammam, A., \& Al-Mutairi, B. (2020). Combined effect of a mixture of silver nanoparticles and calcium hydroxide against Enterococcus faecalis biofilm. Journal of Endodontics, S0099-2399(20)30488-X. Advance online publication. https://doi.org/10.1016/j.joen.2020.07.001

Barros, J., Silva, M. G., Rôças, I. N., Gonçalves, L. S., Alves, F. F., Lopes, M. A., Pina-Vaz, I., \& Siqueira, J. F., Jr (2014). Antibiofilm effects of endodontic sealers containing quaternary ammonium polyethylenimine nanoparticles. Journal of Endodontics, 40(8), 1167-1171. https://doi.org/10.1016/j.joen.2013.12.021

Bystrom, A., Claesson, R., \& Sundqvist, G. (1985). The antibacterial effect of camphorated paramonochlorophenol, camphorated phenol and calcium hydroxide in the treatment of infected root canals. Endodontics \& Dental Traumatology, 1(5), 170-175. https://doi.org/10.1111/j.1600-9657.1985.tb00652.x 
Candeiro, G. T., Correia, F. C., Duarte, M. A., Ribeiro-Siqueira, D. C., \& Gavini, G. (2012). Evaluation of radiopacity, pH, release of calcium ions, and flow of a bioceramic root canal sealer. Journal of Endodontics, 38(6), 842-845. https://doi.org/10.1016/j.joen.2012.02.029

Carvalho, C. N., Freire, L. G., Carvalho, A. P., Duarte, M. A., Bauer, J., \& Gavini, G. (2016). Ions release and ph of calcium hydroxide-, chlorhexidine- and bioactive glass-based endodontic medicaments. Brazilian Dental Journal, 27(3), 325-331. https://doi.org/10.1590/0103-6440201600602

Cheng, X., Qu, T., Ma, C., Xiang, D., Yu, Q., \& Liu, X. (2017). Bioactive mono-dispersed nanospheres with long-term antibacterial effects for endodontic sealing. Journal of Materials Chemistry. B, 5(6), 1195-1204. https://doi.org/10.1039/C6TB02819E

Collares, F. M., Leitune, V., Portella, F. F., Santos, P. D., Balbinot, G. S., Dos Santos, L. A., Parolo, C., \& Samuel, S. (2018). Methacrylate-based root canal sealer containing chlorexidine and $\alpha$-tricalcium phosphate. Journal of Biomedical Materials Research. Part B, Applied biomaterials, 106(4), 1439-1443. https://doi.org/10.1002/jbm.b.33946

de Andrade Ferreira, F. B., Silva E Souza, P., do Vale, M. S., de Moraes, I. G., \& Granjeiro, J. M. (2004). Evaluation of pH levels and calcium ion release in various calcium hydroxide endodontic dressings. Oral Surgery, Oral Medicine, Oral Pathology, Oral Radiology, and Endodontics, 97(3), 388-392. https://doi.org/10.1016/j.tripleo.2003.08.020

Estrela, C., Pécora, J. D., Souza-Neto, M. D., Estrela, C. R., \& Bammann, L. L. (1999). Effect of vehicle on antimicrobial properties of calcium hydroxide pastes. Brazilian Dental Journal, 10(2), 63-72.

Fava, L. R., \& Saunders, W. P. (1999). Calcium hydroxide pastes: classification and clinical indications. International Endodontic Journal, 32(4), 257-282. https://doi.org/10.1046/j.1365-2591.1999.00232.x

Ferreira, F. B., Torres, S. A., Rosa, O. P., Ferreira, C. M., Garcia, R. B., Marcucci, M. C., \& Gomes, B. P. (2007). Antimicrobial effect of propolis and other substances against selected endodontic pathogens. Oral Surgery, Oral Medicine, Oral Pathology, Oral Radiology, and Endodontics, 104(5), 709-716. https://doi.org/10.1016/j.tripleo.2007.05.019

Gao, Y., Jiang, X., Lin, D., Chen, Y., \& Tong, Z. (2016). The starvation resistance and biofilm formation of Enterococcus faecalis in coexistence with Candida albicans, Streptococcus gordonii, Actinomyces viscosus, or Lactobacillus acidophilus. Journal of Endodontics, 42(8), 1233-1238. https://doi.org/10.1016/j.joen.2016.05.002.

Guerreiro, J., Ochoa-Rodrígez, V. M., Rodrigues, E. M., Chavez-Andrade, G. M., Tanomaru-Filho, M., Guerreiro-Tanomaru, J. M., \& Faria, G. (2021). Antibacterial activity, cytocompatibility and effect of Bio-C Temp bioceramic intracanal medicament on osteoblast biology. International Endodontic Journal, 10.1111/iej.13502. Advance online publication. https://doi.org/10.1111/iej.13502

Guerreiro-Tanomaru, J. M., de Faria-Júnior, N. B., Duarte, M. A., Ordinola-Zapata, R., Graeff, M. S., \& Tanomaru-Filho, M. (2013). Comparative analysis of Enterococcus faecalis biofilm formation on different substrates. Journal of Endodontics, 39(3), 346-350. https://doi.org/10.1016/j.joen.2012.09.027

Kangarlou, A., Neshandar, R., Matini, N., \& Dianat, O. (2016). Antibacterial efficacy of AH Plus and AH26 sealers mixed with amoxicillin, triple antibiotic paste and nanosilver. Journal of Dental Research, Dental Clinics, Dental Prospects, 10(4), 220-225. https://doi.org/10.15171/joddd.2016.035

Kim, D., \& Kim, E. (2014). Antimicrobial effect of calcium hydroxide as an intracanal medicament in root canal treatment: a literature review - Part I. In vitro studies. Restorative Dentistry \& Endodontics, 39(4), 241-252. https://doi.org/10.5395/rde.2014.39.4.241

Mohammadi, Z., \& Dummer, P. M. (2011). Properties and applications of calcium hydroxide in endodontics and dental traumatology. International Endodontic Journal, 44(8), 697-730. https://doi.org/10.1111/j.1365-2591.2011.01886.x

Nakajo, K., Komori, R., Ishikawa, S., Ueno, T., Suzuki, Y., Iwami, Y., \& Takahashi, N. (2006). Resistance to acidic and alkaline environments in the endodontic pathogen Enterococcus faecalis. Oral Microbiology and Immunology, 21(5), 283-288. https://doi.org/10.1111/j.1399-302X.2006.00289.x

Nerwich, A., Figdor, D., \& Messer, H. H. (1993). pH changes in root dentin over a 4-week period following root canal dressing with calcium hydroxide. Journal of Endodontics, 19(6), 302-306. https://doi.org/10.1016/s0099-2399(06)80461-9

Oda, D. F., Duarte, M., Andrade, F. B., Moriyama, L. T., Bagnato, V. S., \& de Moraes, I. G. (2019). Antimicrobial action of photodynamic therapy in root canals using LED curing light, curcumin and carbopol gel. International Endodontic Journal, 52(7), 1010-1019. https://doi.org/10.1111/iej.13092

Pereira, T. C., da Silva Munhoz Vasconcelos, L. R., Graeff, M., Ribeiro, M., Duarte, M., \& de Andrade, F. B. (2019). Intratubular decontamination ability and physicochemical properties of calcium hydroxide pastes. Clinical Oral Investigations, 23(3), 1253-1262. https://doi.org/10.1007/s00784-018-2549-0

Peters, C. I., Koka, R. S., Highsmith, S., \& Peters, O. A. (2005). Calcium hydroxide dressings using different preparation and application modes: density and dissolution by simulated tissue pressure. International Endodontic Journal, 38(12), 889-895. https://doi.org/10.1111/j.1365-2591.2005.01035.x

Rehman, K., Saunders, W. P., Foye, R. H., \& Sharkey, S. W. (1996). Calcium ion diffusion from calcium hydroxide-containing materials in endodonticallytreated teeth: an in vitro study. International Endodontic Journal, 29(4), 271-279. https://doi.org/10.1111/j.1365-2591.1996.tb01381.x

Simundi'c Muniti'c M, Poklepovi'c Peri'ci'c T, Utrobi'ci'c A, Bago I, Puljak L (2019) Antimicrobial efficacy of commercially available endodontic bioceramic root canal sealers: a systematic review. PLoS One 14, e0223575.

Swimberghe, R., Coenye, T., De Moor, R., \& Meire, M. A. (2019). Biofilm model systems for root canal disinfection: a literature review. International Endodontic Journal, 52(5), 604-628. https://doi.org/10.1111/iej.13050

Tabrizizadeh, M., Rasti, M., Ayatollahi, F., Mossadegh, M. H., Zandi, H., Dehghan, F., \& Mousavi, Z. (2015). Antimicrobial activity of calcium hydroxide and betamethasone on Enterococcus faecalis; an in vitro assessment. Iranian Endodontic Journal, 10(3), 184-187. https://doi.org/10.7508/iej.2015.03.008

Tanomaru, J. M., Leonardo, M. R., Tanomaru Filho, M., Bonetti Filho, I., \& Silva, L. A. (2003). Effect of different irrigation solutions and calcium hydroxide on bacterial LPS. International Endodontic Journal, 36(11), 733-739. https://doi.org/10.1046/j.1365-2591.2003.00717.x 
Research, Society and Development, v. 10, n. 7, e33310716550, 2021

(CC BY 4.0) | ISSN 2525-3409 | DOI: http://dx.doi.org/10.33448/rsd-v10i7.16550

Villa, N., Santos, V., Costa, U., Mendes, A. T., Duarte, P., Rosa, R., Pereira, J. R., \& Só, M. (2020). A new calcium silicate-based root canal dressing: physical and chemical properties, cytotoxicity and dentinal tubule penetration. Brazilian Dental Journal, 31(6), 598-604. https://doi.org/10.1590/0103-6440202003376

Wang, Z., Shen, Y., \& Haapasalo, M. (2014). Dentin extends the antibacterial effect of endodontic sealers against Enterococcus faecalis biofilms. Journal of Endodontics, 40(4), 505-508. https://doi.org/10.1016/j.joen.2013.10.042

Zancan, R. F., Vivan, R. R., Milanda Lopes, M. R., Weckwerth, P. H., de Andrade, F. B., Ponce, J. B., \& Duarte, M. A. (2016). Antimicrobial activity and physicochemical properties of calcium hydroxide pastes used as intracanal medication. Journal of Endodontics, 42(12), 1822-1828. https://doi.org/10.1016/j.joen.2016.08.017

Zancan, R. F., Canali, L., Tartari, T., Andrade, F. B., Vivan, R. R., \& Duarte, M. (2018). Do different strains of E. faecalis have the same behavior towards intracanal medications in in vitro research? Brazilian Oral Research, 32, e46. https://doi.org/10.1590/1807-3107bor-2018.vol32.0046.

Zmener, O., Pameijer, C. H., \& Banegas, G. (2007). An in vitro study of the pH of three calcium hydroxide dressing materials. Dental Traumatology : official publication of International Association for Dental Traumatology, 23(1), 21-25. https://doi.org/10.1111/j.1600-9657.2005.00447.x. 